\title{
Potential Disease-Modifying Treatment Strategies Targeting Senescence in the Setting of Knee Osteoarthritis
}

\author{
William S Hambright ${ }^{2}$, Austin Yeargan III' ${ }^{1}$, Justin E Hellwinkel ${ }^{2}$, Kaitlyn E Whitney ${ }^{2,4}$, Bailey E Montgomery ${ }^{3}$, \\ Marc J Philippon ${ }^{4}$, Thos A Evans ${ }^{4}$, Bruno Peault ${ }^{5,6}$ and Joseph Buckwalter ${ }^{7}$ and Johnny Huard ${ }^{2 *}$
}

${ }^{1}$ Regenerative Medicine Clinic, Wilmington, USA

${ }^{2}$ Center for Regenerative Sports Medicine, Steadman Philippon Research Institute, USA

${ }^{3}$ Center for Genomic Medicine, Massachusetts General Hospital, USA

${ }^{4}$ The Steadman Clinic, Vail, USA

${ }^{5}$ MRC Centre for Regenerative Medicine, The University of Edinburgh, UK

${ }^{6}$ Orthopaedic Hospital Research Center, David Geffen School of Medicine, University of California at Los Angeles, USA

${ }^{7}$ University of Iowa, Carver College of Medicine, Department of Orthopedics and Rehabilitation, USA

Submission: February 17, 2021; Published: March 17, 2021

*Corresponding author: Johnny Huard, PhD, Chief Scientific Officer, Center for Regenerative Sports Medicine Steadman Philippon Research Institute, 181 West Meadow Drive Suite 1000 Vail, CO 81657, Colorado, USA

\begin{abstract}
The prevalence of osteoarthritis (OA) is increasing and projected to affect one in four adults in the United States by 2040. Limitations associated with recreational and vocational pursuits is expected to increase substantially, resulting in a large impact on individuals and the healthcare system. Conservative treatments for knee $\mathrm{OA}$ have traditionally focused on ameliorating symptoms through non-invasive and minimally invasive procedures. While conservative treatment modalities are considered the "gold standard" in clinical practice, preventative and restorative therapies are desperately needed to improve quality of life in those suffering from symptomatic OA. A large body of literature exists regarding the mechanisms responsible for the pathogenesis of $\mathrm{OA}$ and has enabled the development of alternative treatment modalities, including optimized cell-based therapies (CBTs) and new systemic and intra-articular disease modifying OA drug (DMOAD) approaches. Bone marrow and adipose CBTs offer significant translational advantages in that progenitor cells and bioactive factors can be harvested using minimally invasive technology without the need of in vitro expansion. Enhancing the therapeutic efficacy of these CBTs via senolytic agents also offers a promising new treatment modality. Senolytic agents are considered DMOADs that target senescent cells which release senescenceassociated secretory phenotype (SASP) factors. Attenuating cellular senescence and their secreted constituents via senolytic drugs may offer a novel disease modifying approach for the treatment of OA. In this review, we will briefly summarize OA etiology of the knee, discuss limitations of current treatments of CBTs and DMOADs, and highlight emerging strategies on targeting cellular senescence.
\end{abstract}

Keywords: Osteoarthritis; Orthobiologics; Cell-based therapies; Mesenchymal stromal cells; Senescence; Senescence-associated secretory phenotype

Abbreviations: OA: Osteoarthritis; CBTs: Cell-Based Therapies; DMOADs: Disease modifying osteoarthritis drugs, MSCs: Mesenchymal Stromal Cells; SASP: Senescence-associated Secretory Phenotype

\section{Introduction}

Osteoarthritis $(\mathrm{OA})$ is a progressive degenerative disease of the joint leading to cartilage damage, pain and loss of function [14] affecting an estimated 250 million people worldwide and 27 million people in the United States [5,6]. The knee is the largest synovial joint in the body comprised of synovium, ligaments, avascular cartilage, and different osseous components. Further, the knee joint is a very common site for osteoarthritic pain given the high use and stress subjected to the joint daily [7]. Several important risk factors are associated with $\mathrm{OA}$ onset including but not limited to BMI, previous knee injury, age, gender, family history [8]. OA can be generally categorized into two groups: idiopathic (age-associated OA, AAOA) and secondary, which is 
often after injury or trauma (post-traumatic OA, PTOA) but can also be due to mechanical misalignment. The degeneration of cartilage with OA leads to significant pain and joint dysfunction [2-4]. While causally different, both idiopathic and PTOA share similar symptomology and degenerative drivers that comprise the multi-factorial condition [9].

Currently, there are no effective FDA-approved therapies that are disease modifying interventions to prevent the course of joint destruction due to OA. The most prevalent first-line treatment for $\mathrm{OA}$ is to mitigate pain and restore function with a combination of weight management, physical therapy, mind-body exercises, and analgesia with paracetamol or NSAIDs (topical or oral) [1012]. Another prominent treatment strategy is the use of intraarticular corticosteroids (CS) to reduce pain and inflammation via targeting production of interleukins, leukotrienes, prostaglandins, and metalloproteinases [13-15]. However, the palliative effects of CS for OA are often short-term, can potentially lead to chondral fissuring and promotion of dose-independent structural changes in cartilage, and there are no consistent reports of efficacy [16]. This has resulted in the American Academy of Orthopaedic Surgeons (AAOS) being unable to recommend the use of intraarticular CSs [17]. In addition, surgical approaches exist to repair damaged/degenerated cartilage to mitigate the development or progression of OA. These include bone marrow stimulation, osteochondral allograft transplantation, and autologous chondrocyte implantation that have limitations often requiring total joint replacement which itself is not ideal for younger individuals [18-22] [1,4,23-29].

Several alternative strategies to CS are currently available to consumers but are considered experimental. Cell-based therapies (CBTs), disease-modifying osteoarthritis drugs (DMOADs), or a combination thereof, represent appealing alternatives for the treatment of OA $[13,30]$. More recently, bone marrow and percutaneous adipose tissue CBTs have demonstrated promising short and intermediate term benefits for knee OA [31]. These studies have shown significant improvements in the Visual Analog Scale for Pain, IKDC knee function, Tegner Activity Scale, and Lysholm Knee Scores [32,33]. However, level I evidence of long-term efficacy or significant tissue regeneration is certainly lacking. A critical variable regarding CBT studies has routinely been the discrepancy in the preparation of the orthobiologics product such as the use of point of care devices versus manual preparations in addition to platelet content, platelet activation state, and leukocyte content. One novel and appealing approach for treating age-associated OA (AAOS) and post-traumatic OA (PTOA) is through the local and systemic elimination of senescent cells. Senescent cell burden increases significantly with age and has been shown to promote several age-related pathologies including degenerative joint conditions [34-37]. Senescent cells are non-proliferative, resistant to apoptosis, and secrete a litany of pro-inflammatory factors that promote disease and systemic aging [38]. While the presence of senescent cells in cartilage has been noticed for decades in patients undergoing total knee replacement, the role in OA pathogenesis remains unclear [39-41]. However, recent evidence points towards a potentially causative role in idiopathic and PTOA including findings that demonstrate significant benefits with senescent cell clearance [36,37]. Thus, senescent cells and their paracrine-acting factors likely play a significant role in $\mathrm{OA}$ pathogenesis and has led to several ongoing clinical trials investigating senolytic agents in age-related pathologies, such as frailty (NCT03430037, NCT03675724), Alzheimer's disease, and knee osteoarthritis (NCT03513016, NCT04129944, NCT04210986). Overall, this review provides an overview of key factors involved in OA progression and currently available CBTs and DMOADs for the treatment of OA. We discuss updates to CBTs in the setting of knee OA with a special emphasis on the contributions of senescence to the disease that offer emerging therapeutic targets.

\section{Paradigms for OA Etiology}

\section{Progressive Age-Related Cartilage Degeneration}

$\mathrm{OA}$ is a multifactorial disease that is affected by mechanical derangements (Figure 1) and other etiological factors [42]. Chondrocytes in the extracellular matrix (ECM) are responsive to cyclic loading by clustering along force vectors relative to the biomechanics of gait [43]. Pathologic static and dynamic loading patterns in the axial and appendicular skeleton initiate remodeling of the ECM in which the articular chondrocytes reside [44-46]. This transformation increases the ratio of type one collagen to proteoglycan in the ECM [44-46]. Proteoglycans in the cartilage matrix break down and lose functional capability with escalation of inflammation [44-46]. The resulting ECM construct stiffens and transfers load directly to the subchondral bone [44-46]. Once the physiologic loading capacity exceeds the physical limits of the subchondral bone, pathologic changes of the osteochondral tissues manifest, compromising the elastic modulus of the subchondral bone [47]. Over time, the concentrated force is directed back into the articular surface of the convex side of the joint, where the same process occurs in a more accelerated fashion. Advanced T2 weighted magnetic resonance imaging (MRI) sequences has allowed a clear delineation of these stages of disease and will likely prove helpful in the future when included as a diagnostic tool and an instrument to objectively measure treatment effects.

\section{Trauma Induced Osteoarthritis}

PTOA develops after injury or trauma to the cartilaginous surface caused by impact, shear, and eccentric loading of the joint surface. Sports-related injuries and fractures are the most common mechanisms that predispose to development of PTOA. Furthermore, it is difficult to predict which patients will be predisposed to chronic musculoskeletal issues after trauma. This can be significant for athletes who sustain soft tissue injuries and need to be counseled on their risk for further damage to their joints. Apoptosis and necrosis of chondrocytes secondary to injury is thought to be responsible for degeneration in the acute phase, but perhaps even more significant is the subsequent inflammatory 
phase which affects cartilage at surrounding sites as well [48]. Catabolic inflammation within the joint induces breakdown of cellular components and offsets mechanical stability of the cartilage, making it further susceptible to degeneration. Although
MRI can be a tool to assess cartilage damage, there is currently no modality to predict the rate of degeneration a patient will have in an injured joint.

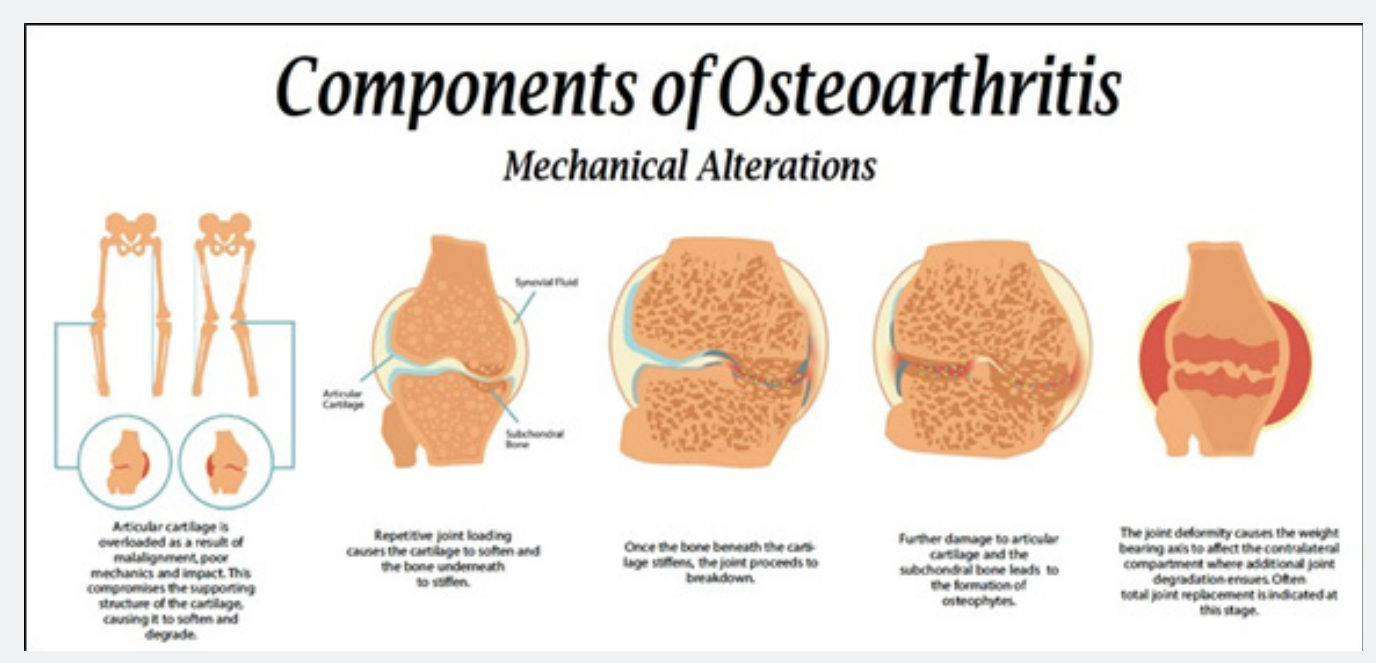

Figure 1: Mechanical induction of the degeneration process in knee OA.

\section{Altered Signaling Pathways in the Pathogenesis of Osteoarthritis}

Repeated cyclic load causes the ECM of articular cartilage and subchondral bone to react through adaptive remodeling and over time becomes mechanically compromised. This is mediated through mechanoreceptors and increased trans-osseous oxygen pressures $\left(\mathrm{PO}_{2}\right)$ favoring catabolic transformation [49]. As the ECM attempts to remodel, altered joint mechanics continue to disrupt the macromolecular network that often results in the activation of proteinases involved in degenerative and inflammatory pathways. With increasing matrix wear, the chondrocytes embedded within the ECM are exposed to shear force and become subject to autolysis [50]. In addition, fragmented proteoglycans and release of intracellular proteins stimulate infiltrating monocytes to the articular lining to induce an inflammatory response (Figure 2). Although cartilage degrading proteins are offset by anabolic cytokines (i.e., interleukin-1 receptor antagonist [IL-1Ra], interleukin-4 [IL-4], interleukin-10 [IL-10], etc.), tissue inhibitors of metalloproteinases (TIMPs), and TNF-stimulated gene 6 (TSG6) HA complexes that limit serine protease activity, [51,52] this balance is shifted towards cartilage degeneration in the setting of OA [53-55].

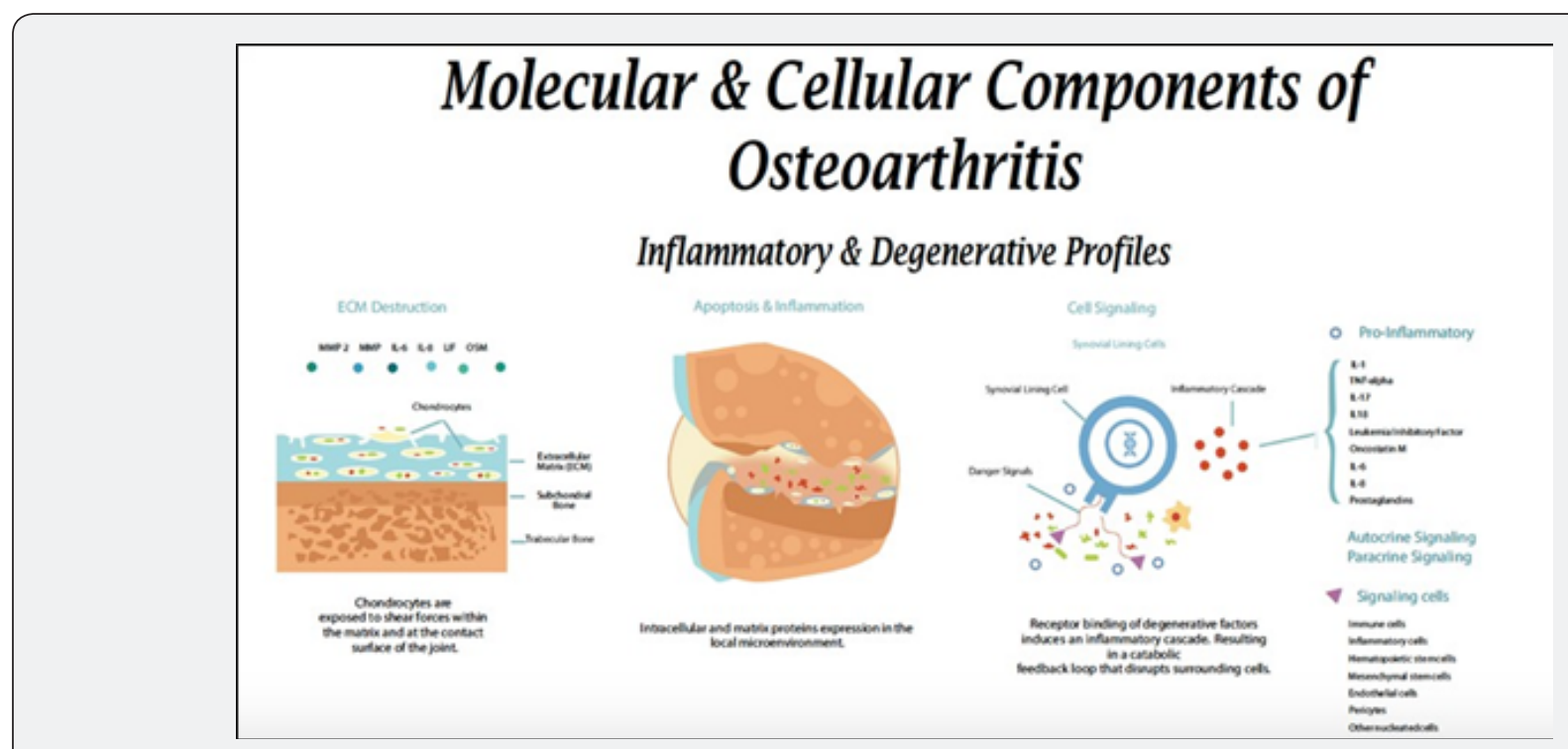

Figure 2: Primary and secondary inflammatory and degenerative cascades in knee OA. 


\section{Orthopedics and Rheumatology Open Access Journal (OROAJ)}

Patient demographics (i.e., age, sex, comorbidities, body mass index [BMI], etc.) are important factors that often guide clinical decisions; however, clinical signs of OA may be considered subclinical in early phases of the disease due to presence of catabolic (degradative) and inflammatory cytokines and chemokines that contribute to the physiopathology. Although it is not clear how these factors are associated with OA-symptomology, there are several major pro-inflammatory mediators that drive degenerative and inflammatory process, including, but not limited to, matrix metalloproteinases (MMPs) $-1,-3,-8,-12$ and -13 , interleukins (ILs) $-1 \beta,-6$, and $-8,-17,-18$, leukemia inhibitory factor (LIF), oncostatin M (OSM), tumor necrosis factor alpha (TNF- $\alpha$ ), and prostaglandins $[53,56]$. In some cases, serial measurements of serum IL- 6 and haptoglobin may provide a baseline assessment and guide treatment [56]. Catabolic biomarkers, such as MMPs, have also been shown to upregulate in synovial fluid in early $\mathrm{OA}$ and establish a negative feedback loop that causes ECM destruction [55,57-59]. While these studies show promise in the diagnostic and prognostic utility of biomarkers for $\mathrm{OA}$, ascertaining periodic chronicity information and biospecimens longitudinally is a major challenge [60]. Given these challenges, there remains a paucity of longitudinal studies that have a common focus on individual biomarkers that may predict incidence or progression of two common OA etiologies (PTOA and age-related OA) [61,62]. In response, several working groups have been established over the last decade to set a framework for the evaluation of joint proteome changes and their impact on the progression of the disease using standardized endpoints [60,62-65]. Continued observations from these working groups are warranted to validate the clinical utility of biomarkers for diagnostic purposes, as well as prognostic purposes following CBT or DMOAD interventions [56,62,64].

\section{Emerging Treatment Strategies for Osteoarthritis}

\section{Cell-based Therapies}

Cell-based therapies (CBTs) can be considered an anabolic (tissue repairing) treatment utilizing a two-component design; a viable nucleated cell component and a soluble protein component (Figure 3). Progenitor cells, namely mesenchymal stem (stromal) cells (MSCs), hematopoietic stem cells (HSCs), and myeloid precursors [66], havebeenused as a treatmentmodality toattenuate OA-symptoms. Specifically, MSCs are capable of self-replication and specialization into many tissue types, including cartilage, bone, tendon, muscle, and fat [67]. MSCs direct tissue responses to injury, stimulate resident stem cells, and prompt apoptosis and cell lysis to initiate a healing response in musculoskeletal tissues. The degree of local inflammation determines whether MSCs respond with a pro- or anti-inflammatory phenotype, which is important when considering their clinical use [68]. Despite their capacity to form clonal cell lines and specialize into tissues of mesodermal lineage, the immunomodulatory and antiinflammatory effects are the most responsible for the clinical experience of pain relief [69], and potentially the most important mechanisms to be studied. A common source of MSCs is from the bone, most often sourced from the iliac crest [70]. However, MSCs makeup only approximately $0.001 \%$ of the bone marrow cell population and have presented hurdles in the clinic including donor site morbidity and pain [71]. In addition, there seems to be variability between content depending on the type of tissue the cells are extracted from and the demographics of the donor [72]. Indeed, more clinical studies are needed to characterize cellular profiles (including MSC surface antigens) to better understand the association between these profiles and clinical outcomes.

\section{Mesenchymal Stem Cells \& Signaling Proteins in
Osteoarthritis}
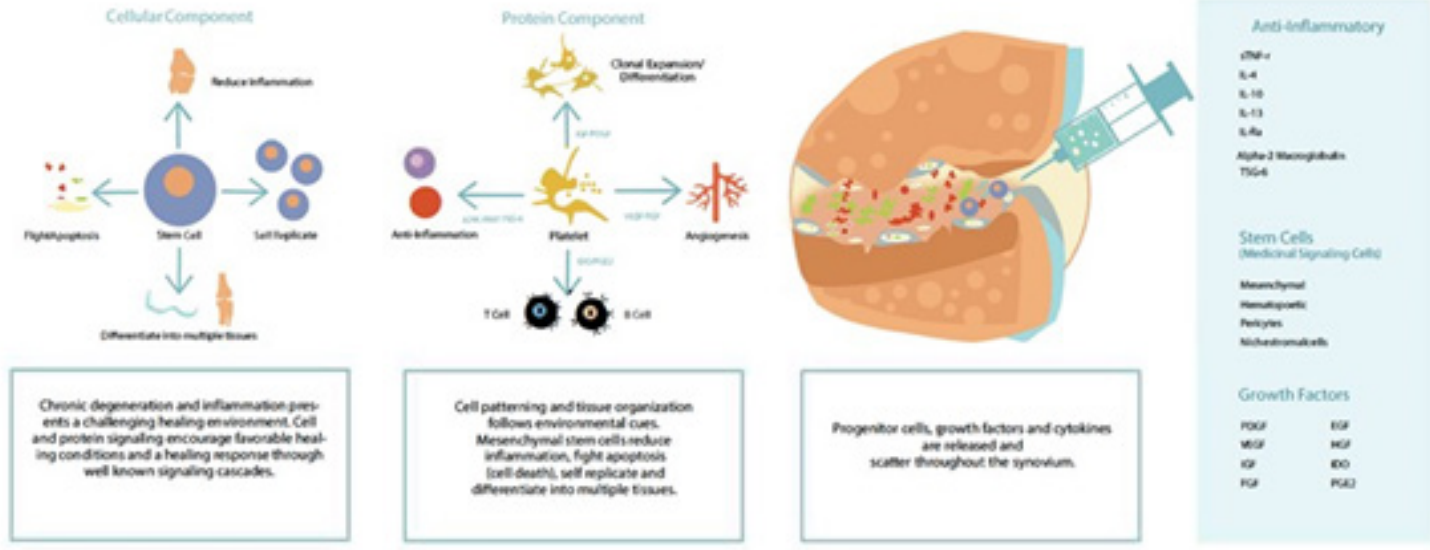

Figure 3: CBT mechanisms of action in the setting of knee OA. 
Due to the limitations of bone marrow CBTs, other cell populations, namely adipose derived stem cells (ADSCs), have garnered interest as of late [73]. These cells, collected from the stromal vascular fraction of adipose tissue lipoaspirate offer a practical advantage in that they are more accessible and provides CD73, CD90, CD105, CD106 positive multi-potent progenitor cells capable of chondrogenic differentiation [74]. Further, ADSCs have demonstrated pro-regenerative immunomodulatory behavior, including the promotion of inhibitory macrophages and $\mathrm{T}$ regulatory cells which may offer additional means to improve clinical symptoms coupled to cartilage regeneration [75]. Indeed, several clinics routinely isolate SVF from lipoaspirate through minimal manipulation practices for various orthopedic indications, including OA. Of note, current evidence suggests that CBTs are limited in efficacy when not considering alterations in joint mechanics. In this setting, it may be most appropriate to perform CBT in early onset OA patients or non-surgical candidates; however, a defined criterion is needed to provide clinical guidance for this subset of patients that are often asymptomatic.

\section{Targeting Cellular Senescence}

Although $\mathrm{OA}$ is thought to be a disease characterized by gradual deterioration of articular cartilage leading to mechanical failure, emerging evidence highlights the importance of cellular dysregulation. Chondrocytes and other joint cells in both AAOA and PTOA are characterized by genomic instability, telomere shortening, dysregulated nutrient sensing, upregulated proinflammatory signaling, mitochondrial dysfunction, and loss of proteostasis [76-78]. Importantly, all these features are hallmarks of cellular senescence and its associated phenotypes, and indeed, senescent chondrocytes, synoviocytes, synovial macrophages have all been found to be present in OA patients [76-78]. Senescence is a cell state defined by loss of proliferative capacity, increased metabolic activity, and importantly, resistance to apoptosis. Senescent cells promote disease and tissue dysfunction via the release of cartilage degrading enzymes and pro-inflammatory mediators otherwise known as the senescence-associated secretory phenotype (SASP) [38]. Cellular senescence can be induced by a variety of extrinsic (mechanical stress, damage signals, inflammatory factors) and intrinsic signals (reactive oxygen species, DNA damage, mitochondrial dysfunction) that leads to the production of a SASP, a collection of various proinflammatory cytokines/chemokines, proteases (MMPs and ADAMTS-4 and -5), and other factors that initiate senescence in neighboring cells and promote disease and tissue dysfunction $[38,79]$.

The role of senescent cells in the pathogenesis of OA has garnered significant interest recently [35]. In support of a causative role for senescent cells in $\mathrm{OA}$, it was demonstrated that injection of senescent cells into the joint capsule of healthy mice was found to induce radiographic and histological evidence of OAlike conditions including severe cartilage degeneration, erosion of femoral condyles, subchondral bone structure alteration, osteophyte formation, and meniscal damage [80]. Inversely, other groups have shown that local clearance of senescent cells within the joint capsule significantly reduces development of injury-induced $\mathrm{OA}$ and promoted a pro-regenerative environment [37]. The use of transgenic mouse models also strongly implicates cellular senescence in driving OA. Using the p16-3MR mouse model, which allows for the visualization of high $\mathrm{p} 16^{\mathrm{INK} 4 \mathrm{a}}$ expressing cells in live animals, it was found that senescent chondrocytes increased dramatically following ACLR-induced OA [37]. This was associated with marked increases in SASP factors, such as MMP-13, IL-6, and IL-1 $\beta$, all of which are known to be largely present in OA $[36,79]$. In INK-ATTAC mice, a slightly different transgenic model that harbors drug inducible senescent cell elimination via caspasedependent apoptosis, it was found that removal of senescent cells late in life could prevent or delay age-related cartilage loss and retain structural integrity of the cartilage like that in young mice [81]. The findings using the p16-3MR and INK-ATTAC models further suggest a role for senescent cells in not only AAOA, but PTOA as well. Senescent cells and their SASP are an important source of pro-inflammatory mediators and pain, which is the most bothersome manifestation of OA for patients. The SASP produced by senescent cells includes cytokines that elicit inflammation (IL6 , TNF- $\alpha$, IL-1 $\beta$ ) but also chemokines (IL-8, CCL5, CCL19) that attract and activate synovial immune cells [36,79]. Following trauma, infiltrating immune cells play an important role in tissue repair through tissue remodeling and debris clearing, which is temporally coordinated via cytokine/chemokine bursts. Thus, SASP factors work in concert with the localized immune response to potentiate or exacerbate repair, fibrosis, or chronic diseases pathogenesis. This is further confounded by the fact that senescent cells likely play a role in normal tissue healing as they accumulate rapidly following injury, attract immune cells necessary for repair, and are subsequently removed by immune cells, namely natural killer cells $[79,82]$. However, with age, senescent cells accumulate and disrupt this balance. The exact SASP factors most responsible for pain in PTOA or AAOA have yet to be fully elucidated, but several pro-inflammatory SASP factors have been linked to pain including IL-1, IL-6, TNF- $\alpha$, and $\mathrm{PGE}_{2} / \mathrm{COX}-2$ [36]. PGE 2 /COX-2 is especially compelling as a mediator of inflammatory pain given $\mathrm{PGE}_{2}$ is known to bind to E prostanoid receptors (EP1-4) which are present in sensory neurons of the peripheral nervous system. Indeed, it has been found that both $\mathrm{PGE}_{2}$ and COX-2 were elevated in the synovium, bone, and meniscus of human OA explants [83]. It has also been recently demonstrated in preliminary studies that oral dosing of senolytic drugs can preserve cartilage proteoglycan content in a progeroid mouse model that exhibits spontaneous OA symptomology, the Zmpste24\% model (unpublished data). Clinically speaking, recent clinical trials have highlighted a potential causal role for cellular senescence in AAOA and PTOA and senescent cells have been found in nearly all tissues of the arthritic joint in preclinical models and in tissues isolated from arthroscopy procedures [39,40,77-79]. Senescent chondrocytes have also been found to be enriched near osteoarthritic lesions, 
strongly suggesting a disease promoting effect on neighboring cells (40). Thus, senescent cells and their associate SASP profiles likely play a role in both the clinical manifestation of OA (pain) and disease pathogenesis (tissue dysfunction and cartilage degradation).

\section{Discussion}

Over the last decade, patient reported results following CBT intervention for the attenuation of OA-symptoms have been increasingly successful. While a great deal of additional research is needed, so far results have been encouraging and many patients have been able to delay or avoid joint replacement surgery and its inherent risks altogether. Although an ideal solution for knee arthritis has yet to present itself, CBTs and DMOADs provide another tool to combat $\mathrm{OA}$ symptoms and potentially treat the underlying disease. However, some reservations should be had. Despite the wide use of CBTs in the field of orthopedics, there are little data on the cost to benefit ratio of biologic treatments due to inconsistencies between providers. In 2009, it was estimated that more than 570 clinics in the U.S. marketed stem-cell therapies for the treatment of various musculoskeletal conditions [84]. The term "stem cell treatment" is an oversimplified label often used to designate treatment with concentrated nucleated marrow cells and selected proteins, including synovial mesenchymal stromal cells, muscle-derived stem cells (MDSCs), adipose-derived stem cells (ADSCs), bone marrow stem cells (BMSCs), peripheral blood stem cells (pericytes). The clinical efficacy of CBTs certainly warrants further study and may call for an education campaign from the AAOS and ABOS to minimize potential patient exploitation $[17,36,79]$. The responsiveness and variability in reported success rates between patients likely reflects our limited understanding of disease pathogenesis and subsequent therapeutic interaction and not the limited efficacy of the CBTs themselves. There are no accepted 'best practices' for regenerative techniques in orthopaedic surgery or published criteria for CBT treatment.

Targeting senescence may offer a novel therapeutic target for the treatment of AAOA and PTOA. In fact, several senolytic compounds that selectively target and inhibit anti-apoptotic pathways in senescent cells have been recently identified and shown to kill senescent cells in vitro and in vivo without affecting quiescent or proliferating cells [35]. Senolytics are a potentially innovative DMOA drug class that is quite appealing given they target senescent cells directly, thereby inducing cell death and abrogating systemic SASP factors [35], with minimal to zero off target effects. There are indeed a few reports demonstrating the efficacy of senolytic drugs in reducing disease phenotypes in PTOA $[36,37,85]$. The overall safety and efficacy of several senolytic drugs to treat chronic diseases have been demonstrated in several preclinical studies and more recently in phase I-II clinical trials for OA. For example, the senolytic drug Dasatinib is an FDA approved drug for leukemia with few side effects while other senolytic drugs like quercetin and fisetin are naturally occurring plant flavonoids tolerable at relatively high doses [35,86,87]. Further, many senolytic compounds target several different antiapoptotic pathways, allowing for a multi-hit approach $[35,86,87]$ and only intermittent administration is likely necessary as only brief disruption of anti-apoptotic pathways is sufficient to kill senescent cells [87]. Cell division dependent resistance is not a concern either for senolytics as senescent cells do not divide and therefore cannot acquire selectively advantageous mutations such as those found in treating cancers or infectious agents. Finally, another benefit to using senolytic drugs is they can be readily incorporated into established clinical practice via intra-articular delivery and are effective via oral administration as well [37].

$\mathrm{OA}$ is a debilitating and costly joint disease that affects millions of individuals each year for which there are currently no available disease modifying therapies [17,36,79]. Identifying new orthobiologic strategies that modulate causal elements of pathology including CBTs from various tissue sources may transform standard of care. While promising, more scientific evidence derived from randomized controlled clinical trials is necessary prior to promotion and widespread use of these therapies. However, DMOADs including senolytic agents may compliment CBTs in combination or as front-line treatment options during prodromal stages of disease (i.e., advanced age). Senolytic drugs may offer a promising new approach for the treatment of not only OA symptoms, but a fundamental driver of pathogenesis, senescent cells and their SASP.

\section{Conclusion}

Osteoarthritis $(\mathrm{OA})$ is an incurable debilitating disease that is the most common joint disease affecting over $10 \%$ of the population aged 60 and older [88]. Few treatment options exist, and most therapies involve analgesics that target symptoms of $\mathrm{OA}$, such as pain and swelling. Thus, preventative, and restorative therapies are desperately needed to improve quality of life. Cellbased therapies such as concentrated bone marrow aspirate take advantage of autologous mesenchymal stromal cells (MSCs) and offer a uniquely promising disease modifying treatment modality to reduce catabolic processes degenerating articular cartilage and subchondral bone deterioration, reduce pain, and/or restore motion and function. However, the quantity and relative makeup of the pro-regenerative factors and cells within bone marrow aspirate concentrate that drive positive clinical outcomes is currently unknown with significant variance in clinical efficacy. Resident senescent cells and their tissue degrading SASP factors are prominent in various joint tissues and likely promote agerelated and trauma induced OA. Pharmacologically targeting senescence and the SASP via senolytic agents thus offers a novel therapeutic approach for OA. Indeed, targeted elimination of senescence cells in preclinical studies has been shown to reduce OA symptomology [89] while the injection of senescent cells into knee joints of mice exacerbates OA symptomology [80]. Current randomized controlled clinical studies are also underway by our 
group and others to investigate the efficacy of senotherapeutic agents through oral administration (NCT03513016, NCT04129944, NCT04210986). These studies include patient reported measurements of pain and function (IKDC, Lysholm, TEGNER, WOMAC), radiographic grading (Kellgren Lawrence), MRI assessment of cartilage health (T2 mapping), biomarker assessment of peripheral blood and bone marrow aspirate concentrate. Other outcome measures include the evaluation of senescence indices such as serum and bone marrow SASP markers, senescence quantification in peripheral blood mononuclear cells (PBMCs), and senescence related transcript levels of PBMCs. Other delivery modalities are also an option for senolytic therapy including intra-articular injection and potentially to even to treat orthobiologic products directly either intraoperatively or during processing prior to autologous administration. Most importantly, because senescent cell accumulation and chronic sterile inflammation are fundamental properties of aging, senotherapies may act as Disease-modifying OA drugs (DMOADs) in the context of idiopathic and trauma induced OA.

\section{References}

1. O'Driscoll SW (1998) The healing and regeneration of articular cartilage. J Bone Joint Surg Am 80(12): 1795-1812.

2. Bentley G, Biant LC, Carrington RW, Akmal M, Goldberg A, et al. (2003) A prospective, randomised comparison of autologous chondrocyte implantation versus mosaicplasty for osteochondral defects in the knee. J Bone Joint Surg Br 85(2): 223-230.

3. Easton BT (2001) Evaluation and treatment of the patient with osteoarthritis. J Fam Pract 50(9): 791-797.

4. LaPrade RF, Swiontkowski MF (1999) New horizons in the treatment of osteoarthritis of the knee. JAMA 281(10): 876-878.

5. da Costa BR, Hari R, Juni P (2016) Intra-articular Corticosteroids for Osteoarthritis of the Knee. JAMA 316(24): 2671-2672.

6. Nelson AE, Allen KD, Golightly YM, Goode AP, Jordan JM (2014) A systematic review of recommendations and guidelines for the management of osteoarthritis: The chronic osteoarthritis management initiative of the U.S. bone and joint initiative. Semin Arthritis Rheum 43(6): 701-712.

7. Richebe P, Capdevila X, Rivat C (2018) Persistent Postsurgical Pain: Pathophysiology and Preventative Pharmacologic Considerations. Anesthesiology 129(3): 590-607.

8. Berenbaum F (2013) Osteoarthritis as an inflammatory disease (osteoarthritis is not osteoarthrosis!). Osteoarthritis Cartilage 21(1): 16-21.

9. Mora JC, Przkora R, Cruz-Almeida Y (2018) Knee osteoarthritis: pathophysiology and current treatment modalities. J Pain Res 11: 2189-2196.

10. Bannuru RR, Osani MC, Vaysbrot EE, Arden NK, Bennell K, et al. (2019) OARSI guidelines for the non-surgical management of knee, hip, and polyarticular osteoarthritis. Osteoarthritis Cartilage 27(11): 15781589.

11. Bijlsma JW, Berenbaum F, Lafeber FP (2011) Osteoarthritis: an update with relevance for clinical practice. Lancet 377(9783): 2115-2126.

12. Wu Y, Goh EL, Wang D, Ma S (2018) Novel treatments for osteoarthritis: an update. Open Access Rheumatol 10: 135-140.

13. Ayhan E, Kesmezacar H, Akgun I (2014) Intraarticular injections (corticosteroid, hyaluronic acid, platelet rich plasma) for the knee osteoarthritis. World J Orthop 5(3): 351-361.

14. Dulay GS, Cooper C, Dennison EM (2015) Knee pain, knee injury, knee osteoarthritis \& work. Best Pract Res Clin Rheumatol 29(3): 454-461.

15. Richards MM, Maxwell JS, Weng L, Angelos MG, Golzarian J (2016) Intraarticular treatment of knee osteoarthritis: from anti-inflammatories to products of regenerative medicine. Phys Sportsmed 44(2): 101-108.

16. Zeng C, Lane NE, Hunter DJ, Wei J, Choi HK, et al. (2019) Intra-articular corticosteroids and the risk of knee osteoarthritis progression: results from the Osteoarthritis Initiative. Osteoarthritis Cartilage 27(6): 855862.

17. Surgeons AAoO (2017) Management of Osteoarthritis of the Hip: Evidence-Based Clinical Practice Guideline 2017: 106 - 208.

18. Gossec L, Dougados M (2004) Intra-articular treatments in osteoarthritis: from the symptomatic to the structure modifying. Ann Rheum Dis 63(5): 478-482.

19. Johnson LL (2001) Arthroscopic abrasion arthroplasty: a review. Clin Orthop Relat Res 2001(391 Suppl): S306-S317.

20. Steadman JR, Rodkey WG, Rodrigo JJ (2001) Microfracture: surgical technique and rehabilitation to treat chondral defects. Clin Orthop Relat Res 2001(391 Suppl): S362-S369.

21. Binette F, McQuaid DP, Haudenschild DR, Yaeger PC, McPherson JM, et al. (1998) Expression of a stable articular cartilage phenotype without evidence of hypertrophy by adult human articular chondrocytes in vitro. J Orthop Res 16(2): 207-216.

22. Giannoni P, Pagano A, Maggi E, Arbico R, Randazzo N, et al. (2005) Autologous chondrocyte implantation (ACI) for aged patients: development of the proper cell expansion conditions for possible therapeutic applications. Osteoarthritis Cartilage 13(7): 589-600.

23. Archibeck MJ, White RE Jr (2003) What's new in adult reconstructive knee surgery. J Bone Joint Surg Am 85(7): 1404-1411.

24. Felson DT, Lawrence RC, Hochberg MC, McAlindon T, Dieppe PA, et al. (2000) Osteoarthritis: new insights. Part 2: treatment approaches. Ann Intern Med 133(9): 726-737.

25. Hangody L, Kish G, Karpati Z, Szerb I, Udvarhelyi I (1997) Arthroscopic autogenous osteochondral mosaicplasty for the treatment of femoral condylar articular defects. A preliminary report. Knee Surg Sports Traumatol Arthrosc 5(4): 262-267.

26. Huo MH, Javad Parvizi, Nathan F Gilbert (2002) What's new in hip arthroplasty. J Bone Joint Surg Am 84(10): 1894-1905.

27. Kearon C (2003) Duration of venous thromboembolism prophylaxis after surgery. Chest. 2003;124(6 Suppl): 386S-92S.

28. Robert H (2011) Chondral repair of the knee joint using mosaicplasty. Orthop Traumatol Surg Res 97(4): 418-429.

29. Turpie AG (2002) Optimizing prophylaxis of venous thromboembolism. Semin Thromb Hemost 28 Suppl 2: 25-32.

30. Whitney KE, Liebowitz A, Bolia IK, Chahla J, Ravuri S, et al. (2017) Current perspectives on biological approaches for osteoarthritis. Ann N Y Acad Sci 1410(1): 26-43.

31. Dubey NK, Mishra VK, Dubey R, Syed-Abdul S, Wang JR, et al. (2018) Combating Osteoarthritis through Stem Cell Therapies by Rejuvenating Cartilage: A Review. Stem Cells Int 2018: 5421019.

32. Awad ME, Hussein KA, Helwa I, Abdelsamid MF, Aguilar-Perez A, et al. (2019) Meta-Analysis and Evidence Base for the Efficacy of Autologous Bone Marrow Mesenchymal Stem Cells in Knee Cartilage Repair: Methodological Guidelines and Quality Assessment. Stem Cells Int 2019: 3826054. 
33. Cavallo C, Boffa A, Andriolo L, Silva S, Grigolo B, et al. (2021) Bone marrow concentrate injections for the treatment of osteoarthritis: evidence from preclinical findings to the clinical application. Int Orthop 45(2): 525-538.

34. Gao SG, Zeng C, Li LJ, Luo W, Zhang FJ, et al. (2016) Correlation between senescence-associated beta-galactosidase expression in articular cartilage and disease severity of patients with knee osteoarthritis. Int J Rheum Dis 19(3): 226-232.

35. Kirkland JL, Tchkonia T, Zhu Y, Niedernhofer LJ, Robbins PD (2017) The Clinical Potential of Senolytic Drugs. J Am Geriatr Soc 65(10): 2297 2301.

36. Jeon OH, David N, Campisi J, Elisseeff JH (2018) Senescent cells and osteoarthritis: a painful connection. J Clin Invest 128(4): 1229-1237.

37. Jeon OH, Kim C, Laberge RM, Demaria M, Rathod S, et al. (2017) Local clearance of senescent cells attenuates the development of posttraumatic osteoarthritis and creates a pro-regenerative environment. Nat Med 23(6): 775-781.

38. Coppe JP, Desprez PY, Krtolica A, Campisi J (2010) The senescenceassociated secretory phenotype: the dark side of tumor suppression. Annu Rev Pathol 5: 99-118.

39. Martin JA, Buckwalter JA (2002) Human chondrocyte senescence and osteoarthritis. Biorheology 39(1-2): 145-52.

40. Martin JA, Buckwalter JA (2003) The role of chondrocyte senescence in the pathogenesis of osteoarthritis and in limiting cartilage repair. J Bone Joint Surg Am 85-A Suppl 2: 106-110.

41. Mollano AV, Martin JA, Buckwalter JA (2002) Chondrocyte senescence and telomere regulation: implications in cartilage aging and cancer (a brief review). Iowa Orthop J 22: 1-7.

42. Yeargan Iii A, Md I (2018) Knee Arthritis is a Mechanically Induced Disease with Predictably Sinister Molecular Consequences Bone marrow signaling cell concentrates and molecular treatment modalities in the setting of orthopedic surgical manifestations of musculoskeletal disease: Arthritic knee pain.

43. Kumar D, Manal KT, Rudolph KS (2013) Knee joint loading during gait in healthy controls and individuals with knee osteoarthritis. Osteoarthritis Cartilage 21(2): 298-305.

44. Cox TR, Erler JT (2011) Remodeling and homeostasis of the extracellular matrix: implications for fibrotic diseases and cancer. Dis Model Mech 4(2): 165-178.

45. Maldonado M, Nam J (2013) The role of changes in extracellular matrix of cartilage in the presence of inflammation on the pathology of osteoarthritis. Biomed Res Int 2013: 284873.

46. Musumeci G (2016) The Effect of Mechanical Loading on Articular Cartilage. J Rheumatol pp. 154-161.

47. Stewart HL, Kawcak CE (2018) The Importance of Subchondral Bone in the Pathophysiology of Osteoarthritis. Front Vet Sci 5: 178.

48. Carbone A, Rodeo S (2017) Review of current understanding of posttraumatic osteoarthritis resulting from sports injuries. J Orthop Res 35(3): 397-405.

49. Blalock D, Miller A, Tilley M, Wang J (2015) Joint instability and osteoarthritis. Clin Med Insights Arthritis Musculoskelet Disord 8: 1523.

50. Vincent KR, Conrad BP, Fregly BJ, Vincent HK (2012) The pathophysiology of osteoarthritis: a mechanical perspective on the knee joint. PM R 4(5 Suppl): S3-9.

51. Chamberlain CS, Leiferman EM, Frisch KE, Brickson SL, Murphy WL, et al. (2013) Interleukin expression after injury and the effects of interleukin-1 receptor antagonist. PLoS One 8(8): e71631.

52. Chamberlain CS, Leiferman EM, Frisch KE, Wang S, Yang X, et al. (2011) The influence of interleukin-4 on ligament healing. Wound Repair Regen 19(3): 426-435.

53. Blasioli DJ, Kaplan DL (2014) The roles of catabolic factors in the development of osteoarthritis. Tissue Eng Part B Rev 20(4): 355-363.

54. Lieberthal J, Sambamurthy N, Scanzello CR (2015) Inflammation in joint injury and post-traumatic osteoarthritis. Osteoarthritis Cartilage 23(11): 1825-1834

55. Mueller MB, Tuan RS (2011) Anabolic/Catabolic balance in pathogenesis of osteoarthritis: identifying molecular targets. PM R 3(6 Suppl 1): S3-11.

56. Liao W, Li Z, Li T, Zhang Q Zhang H, et al. (2018) Proteomic analysis of synovial fluid in osteoarthritis using SWATHmass spectrometry. Mol Med Rep 17(2): 2827-2836.

57. Julovi SM, Yasuda T, Shimizu M, Hiramitsu T, Nakamura T (2004) Inhibition of interleukin-1beta-stimulated production of matrix metalloproteinases by hyaluronan via CD44 in human articular cartilage. Arthritis Rheum 50(2): 516-525.

58. Wang CT, Lin YT, Chiang BL, Lin YH, Hou SM (2006) High molecular weight hyaluronic acid down-regulates the gene expression of osteoarthritis-associated cytokines and enzymes in fibroblast-like synoviocytes from patients with early osteoarthritis. Osteoarthritis Cartilage 14(12): 1237-1247.

59. Li H, Wang D, Yuan Y, Min J (2017) New insights on the MMP-13 regulatory network in the pathogenesis of early osteoarthritis. Arthritis Res Ther 19(1): 248.

60. Kraus VB, Hargrove DE, Hunter DJ, Renner JB, Jordan JM (2017) Establishment of reference intervals for osteoarthritis-related soluble biomarkers: the FNIH/OARSI OA Biomarkers Consortium. Ann Rheum Dis. 76(1): 179-185.

61. Watt FE, Corp N, Kingsbury SR, Frobell R, Englund M, Felson DT, et al. (2019) Towards prevention of post-traumatic osteoarthritis: report from an international expert working group on considerations for the design and conduct of interventional studies following acute knee injury. Osteoarthritis Cartilage 27(1): 23-33.

62. Kraus VB, Burnett B, Coindreau J, Cottrell S, Eyre D, et al. (2011) Application of biomarkers in the development of drugs intended for the treatment of osteoarthritis. Osteoarthritis Cartilage 19(5): 515542 .

63. Bauer DC, Hunter DJ, Abramson SB, Attur M, Corr M, et al. (2006) Classification of osteoarthritis biomarkers: a proposed approach. Osteoarthritis Cartilage 14(8): 723-727.

64. Bay-Jensen AC, Reker D, Kjelgaard-Petersen CF, Mobasheri A, Karsdal MA, et al. (2016) Osteoarthritis year in review 2015: soluble biomarkers and the BIPED criteria. Osteoarthritis Cartilage 24(1): 9-20.

65. Mobasheri A, Bay-Jensen AC, van Spil WE, Larkin J, Levesque MC (2017) Osteoarthritis Year in Review 2016: biomarkers (biochemical markers). Osteoarthritis Cartilage 25(2): 199-208.

66. Chahla J, Mannava S, Cinque ME, Geeslin AG, Codina D, et al. (2017) Bone Marrow Aspirate Concentrate Harvesting and Processing Technique. Arthrosc Tech 6(2): e441-e445.

67. Caplan AI (2007) Adult mesenchymal stem cells for tissue engineering versus regenerative medicine. J Cell Physiol 213(2): 341-347.

68. Waterman RS, Henkle SL, Betancourt AM (2012) Mesenchymal stem cell 1 (MSC1)-based therapy attenuates tumor growth whereas MSC2treatment promotes tumor growth and metastasis. PLoS One 7(9): e45590. 
69. Kay AG, Long G, Tyler G, Stefan A, BroadfootSJ, etal. (2017) Mesenchymal Stem Cell-Conditioned Medium Reduces Disease Severity and Immune Responses in Inflammatory Arthritis. Sci Rep 7(1): 18019.

70. Hyer CF, Berlet GC, Bussewitz BW, Hankins T, Ziegler HL, et al. (2013) Quantitative assessment of the yield of osteoblastic connective tissue progenitors in bone marrow aspirate from the iliac crest, tibia, and calcaneus. J Bone Joint Surg Am 95(14): 1312-1316.

71. Sugaya H, Yoshioka T, Kato T, Taniguchi Y, Kumagai H, et al. (2018) Comparative Analysis of Cellular and Growth Factor Composition in Bone Marrow Aspirate Concentrate and Platelet-Rich Plasma. Bone Marrow Res 2018: 1549826.

72. Ziegler CG, Van Sloun R, Gonzalez S, Whitney KE, DePhillipo NN, et al. (2019) Characterization of Growth Factors, Cytokines, and Chemokines in Bone Marrow Concentrate and Platelet-Rich Plasma: A Prospective Analysis. Am J Sports Med 2019: 363546519832003.

73. Frese L, Dijkman PE, Hoerstrup SP (2016) Adipose Tissue-Derived Stem Cells in Regenerative Medicine. Transfus Med Hemother 43(4): 268-274.

74. Hurley ET, Yasui Y, Gianakos AL, Seow D, Shimozono Y, et al. (2018) Limited evidence for adipose-derived stem cell therapy on the treatment of osteoarthritis. Knee Surg Sports Traumatol Arthrosc 26(11): 3499-3507.

75. Mei L, Shen B, Ling P, Liu S, Xue J, et al. (2017) Culture-expanded allogenic adipose tissue-derived stem cells attenuate cartilage degeneration in an experimental rat osteoarthritis model. PLoS One 12(4): e0176107.

76. Loeser RF (2009) Aging and osteoarthritis: the role of chondrocyte senescence and aging changes in the cartilage matrix. Osteoarthritis Cartilage 17(8): 971-979.

77. Martin JA, Brown T, Heiner A, Buckwalter JA (2004) Post-traumatic osteoarthritis: the role of accelerated chondrocyte senescence. Biorheology 41(3-4): 479-491.

78. Martin JA, Brown TD, Heiner AD, Buckwalter JA (2004) Chondrocyte senescence, joint loading and osteoarthritis. Clin Orthop Relat Res (427 Suppl): S96-103.
79. McCulloch K, Litherland GJ, Rai TS (2017) Cellular senescence in osteoarthritis pathology. Aging Cell 16(2): 210-218.

80. Xu M, Bradley EW, Weivoda MM, Hwang SM, Pirtskhalava T, et al. (2017) Transplanted Senescent Cells Induce an Osteoarthritis-Like Condition in Mice. J Gerontol A Biol Sci Med Sci 72(6): 780-785.

81. Baker DJ, Childs BG, Durik M, Wijers ME, Sieben CJ, et al. (2016) Naturally occurring p16(Ink4a)-positive cells shorten healthy lifespan. Nature 530(7589): 184-189.

82. Krizhanovsky V, Yon M, Dickins RA, Hearn S, Simon J, et al. (2008) Senescence of activated stellate cells limits liver fibrosis. Cell 134(4): 657-667.

83. Sato T, Konomi K, Fujii R, Aono H, Aratani S, et al. (2011) Prostaglandin EP2 receptor signalling inhibits the expression of matrix metalloproteinase 13 in human osteoarthritic chondrocytes. Ann Rheum Dis 70(1): 221-226.

84. Kotlarz H, Gunnarsson CL, Fang H, Rizzo JA (2009) Insurer and out-ofpocket costs of osteoarthritis in the US: evidence from national survey data. Arthritis Rheum 60(12): 3546-3553.

85. Zheng W, Feng Z, You S, Zhang H, Tao Z, et al. (2017) Fisetin inhibits IL-1beta-induced inflammatory response in human osteoarthritis chondrocytes through activating SIRT1 and attenuates the progression of osteoarthritis in mice. Int Immunopharmacol 45: 1351-1347.

86. Schafer MJ, White TA, Iijima K, Haak AJ, Ligresti G, et al. (2017) Cellular senescence mediates fibrotic pulmonary disease. Nat Commun 8: 14532.

87. Zhu Y, Tchkonia T, Pirtskhalava T, Gower AC, Ding H, et al. (2015) The Achilles' heel of senescent cells: from transcriptome to senolytic drugs. Aging Cell 14(4): 644-658.

88. Zhang Y, Jordan JM (2010) Epidemiology of osteoarthritis. Clin Geriatr Med 26(3): 355-369.

89. Chang J, Wang Y, Shao L, Laberge RM, Demaria M, et al. (2016) Clearance of senescent cells by ABT263 rejuvenates aged hematopoietic stem cells in mice. Nat Med 22(1): 78-83.

\section{Your next submission with Juniper Publishers will reach you the below assets}

- Quality Editorial service

- Swift Peer Review

- Reprints availability

- E-prints Service

- Manuscript Podcast for convenient understanding

- Global attainment for your research

- Manuscript accessibility in different formats

( Pdf, E-pub, Full Text, Audio)

- Unceasing customer service

Track the below URL for one-step submission

https://juniperpublishers.com/online-submission.php 